\title{
Un análisis dinámico de la calidad del trabajo en España. Los efectos de la crisis económica
}

\author{
MARIA CRUZ MERINO LLORENTE \\ Departamento Economía Aplicada, UNIVERSIDAD DE VALLADOLID, ESPAÑA. E-mail: \\ momerino@ea.uva.es
}

NOELIA SOMARRIBA ARECHAVALA

Departamento Economía Aplicada, UNIVERSIDAD DE VALLADOLID, ESPAÑA. E-mail: nsomarri@eco.uva.es

ANA MARÍA NEGRO MACHO

Departamento Sociología y Trabajo Social, UNIVERSIDAD DE VALLADOLID, ESPAÑA. E-mail: amnegro@trs.uva.es

\begin{abstract}
RESUMEN
Entre los principales efectos de la actual crisis económica en España se encuentra la gran destrucción de puestos de trabajo, superando actualmente la tasa de paro el $22 \%$. Ante esta situación, las actuaciones de los poderes públicos se encaminan hacia la recuperación económica y, así la generación de empleo, incidiendo en la cantidad y no tanto en la calidad laboral.

El objetivo de este artículo es medir la calidad del empleo en las Comunidades Autónomas españolas antes y durante la crisis, utilizando el método de la distancia $\mathrm{P}_{2}$ para componer un Indicador Sintético de la Calidad del Trabajo Dinámico entre 2000 y 2010. Los resultados del estudio muestran un empeoramiento de la calidad laboral en las distintas Comunidades durante el período analizado acompañado de un incremento de la polarización entre regiones en cuanto a los niveles de la calidad del empleo.
\end{abstract}

Palabras clave: Calidad del trabajo, Indicadores Sintéticos, Medidas de Distancia.

\section{A Dynamic Analysis of the Quality of Work in Spain. The Effects of the Economic Crisis}

\begin{abstract}
Among the main effects of the current economic crisis in Spain is the great loss of jobs, the unemployment rate exceeding $22 \%$. In this situation, the actions of government aim to begin economic recovery and so create jobs. It affects the work quantity and not quality.

The objective of this article is to measure the employment quality in the Spanish regions before and after the crisis. To achieve this goal, we use the $\mathrm{P}_{2}$ distance method to compose a Synthetic Dynamics Indicator of Quality of Work from 2000 to 2010. The study results show a deterioration in job quality in the different regions during the period accompanied by an increase in spatial polarization in terms of levels of job quality.
\end{abstract}

Keywords: Work Quality, Synthetics Indicators, Distance Measure.

Clasificación JEL: J81, C43

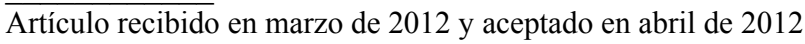

Artículo disponible en versión electrónica en la página www.revista-eea.net, ref. ə-30112 


\section{INTRODUCCIÓN}

En el actual escenario de crisis económica, en el que los políticos centran sus objetivos en el logro de mayores tasas de empleo, como mecanismo para iniciar el crecimiento económico y garantizar el estado de bienestar de los ciudadanos, el estudio de la calidad del trabajo ${ }^{1}$ cobra un especial interés. Aunque el logro de altas tasas de empleo es importante, no podemos obviar las repercusiones que la ausencia de calidad en los puestos de trabajo tiene sobre los individuos y la sociedad. Por ello, en el presente trabajo se propone un estudio de la calidad laboral en España a nivel de las Comunidades Autónomas durante la última década, en concreto para los años 2000, 2007 y 2010. La selección de estos instantes temporales se ha hecho con el objetivo de contrastar si las distintas fases del ciclo económico afectan a los niveles de calidad del trabajo por regiones.

En concreto, en este trabajo nos proponemos dar respuesta a preguntas como: ¿ha empeorado el nivel de la calidad del trabajo en España?, ¿qué Comunidades se han visto más perjudicadas por los efectos de la crisis y en qué medida? o ¿se han acentuado las diferencias entre las Comunidades?

Para llevar a cabo el análisis se propone el diseño de un Indicador Sintético de Calidad del Trabajo Dinámico (en adelante, ISCTD) para lo que utilizaremos la medida de distancia $\mathrm{P}_{2}$. Una de las posibilidades que ofrece el uso de esta metodología es la realización de análisis temporales, siempre que la distancia entre los períodos de tiempo objeto de comparación no sea muy grande, como es el caso que nos ocupa, de forma que esta comparación tenga sentido. Disponemos, así, de una herramienta que nos permite analizar la evolución de la calidad del trabajo desde una óptica temporal. Además esta metodología presenta como ventaja que resuelve de forma idónea algunos de los problemas más habituales que se plantean a la hora de elaborar indicadores sintéticos, entre los que merece destacarse el de la agregación y el de la ponderación de los indicadores simples.

Por otro lado, se empleará en el diseño del ISCTD información, tanto de carácter objetivo como subjetivo, este último reflejará las percepciones que los trabajadores tienen sobre su trabajo y el entorno que le rodea (Somarriba et al. 2010). Sin duda alguna, la incorporación de este tipo de variables enriquecerá de forma sustancial nuestro análisis, ofreciendo una perspectiva diferente a la de los estudios tradicionales sobre el tema.

Después de esta introducción, en un primer apartado se realiza una panorámica de la situación del mercado de trabajo español en el contexto de la Unión Europea ante la actual crisis económica. En el siguiente epígrafe se define el concepto de calidad del trabajo en el que se sustentan las mediciones. A conti-

\footnotetext{
${ }^{1}$ En adelante, calidad del empleo y calidad del trabajo se utilizarán como conceptos sinónimos.
} 
nuación, se efectúa una presentación de la metodología de la medida de distancia $\mathrm{P}_{2}$ (en adelante, $\mathrm{DP}_{2}$ ). Y posteriormente, se procede a analizar los resultados de nuestro indicador sintético de calidad del trabajo dinámico y de sus componentes, finalizando con las conclusiones y una serie de reflexiones.

\section{EL MERCADO DE TRABAJO ESPAÑOL Y LA CRISIS ECONÓMICA}

La fuerte desaceleración económica experimentada en los países de la Unión Europea $^{2}$ y a nivel internacional, con un claro repunte del número de parados, ha centrado el debate sobre la cantidad de empleo, dejando a un lado su calidad. Si en la Estrategia de Lisboa (2000) la Comisión Europea hablaba de "crear más y mejores empleos", en 2010 la Estrategia Europa $2020^{3}$ incide en la creación de empleo, en fomentar una economía que promueva la cohesión económica, social y territorial, marcándose como objetivo el logro de una tasa de empleo del $75 \%$ de la población de entre 20 y 64 años en 2020, objetivo bastante ambicioso pensado básicamente para garantizar la sostenibilidad del modelo social y del sistema de bienestar existente. Se sigue poniendo énfasis en las políticas de flexiguridad, como mecanismo para elevar las tasas de actividad y facilitar las transiciones en el mercado laboral, considerando primordial el papel de la formación permanente.

La Agenda de nuevas cualificaciones y empleos, una de las iniciativas de la Estrategia 2020, tiene entre sus prioridades mejorar la calidad del empleo y las condiciones de trabajo. Aunque en esta materia se ha progresado, todavía queda bastante camino por recorrer. Por lo tanto, interesa avanzar en el logro de la calidad laboral porque además de ser buena en sí misma, se observa que los países que se preocupan por esta cuestión son los que presentan mejores resultados en los niveles de empleo y en la productividad de sus empresas. La Comisión Europea reconoce que en estos años de crisis una buena parte de los puestos de trabajo ha sufrido un deterioro de sus condiciones de trabajo ${ }^{4}$ y se ha producido un mayor flujo de transiciones en el mercado laboral. Para alcanzar el objetivo

\footnotetext{
${ }^{2}$ En 2010 ya se observan signos de recuperación en determinados Estados miembros de la Unión Europea, que continúan en 2011, en especial en Alemania, Suecia o Austria.

${ }^{3}$ Estrategia europea a largo plazo con cinco objetivos y siete iniciativas, aprobada por el Consejo Europeo en 2010, para salir fortalecidos de la crisis y convertir a la Unión Europea en una economía inteligente, sostenible e integradora (sus tres grandes prioridades).

${ }^{4}$ Siguiendo a Green (2009), a medida que una economía se hace más opulenta, sus ciudadanos son más ricos y pueden utilizar esa riqueza en "comprar" mejores condiciones de trabajo y más seguras. En los últimos años en el Reino Unido a pesar de un aumento de las ocupaciones no manuales cualificadas, que se podrían calificar de buenos puestos de trabajo, se observa cierto deterioro de distintos aspectos de la calidad del empleo, entre ellos una intensificación del trabajo en general.
} 
del 2020 las políticas de empleo a favor de la creación de puestos de trabajo de calidad tienen un gran papel. El logro de un buen entorno laboral es esencial para el impulso del potencial de la mano de obra y un elemento clave de competitividad.

Durante el período de nuestro estudio, 2000-2010, la economía española ha pasado de estar en una situación de gran crecimiento a sufrir el impacto de una grave crisis en la que todavía se encuentra inmersa. Si entre 2000 y 2007 se crearon en España casi cinco millones de empleos (4.927.100), en sólo menos de la mitad de tiempo se han destruido casi el $40 \%$ de esos puestos (1.890.400), lo que ha supuesto un retroceso en la convergencia iniciada hacia los objetivos de Lisboa del 2010 y una subida de la tasa de paro en más de 12 puntos porcentuales entre esos años (del 7,95\% al 20,09\%) $)^{5}$, como consecuencia tanto de la reducción del empleo como del aumento de la población activa ${ }^{6}$.

Nuestra economía se caracteriza, a diferencia de otros países, por su patrón cíclico, esto es, una gran sensibilidad de creación y destrucción de empleo según las fases del ciclo económico, fundamentalmente porque el ajuste se hace recurriendo al empleo, lo que conlleva un elevado coste social en los períodos recesivos. Ajuste que es favorecido por la contratación temporal y por la escasa incidencia de mecanismos de flexibilidad interna en las empresas. En España se han generado un elevado número de puestos de trabajo temporales, de muy corta duración ${ }^{7}$. Teniendo en cuenta esto, la mejora de las cualificaciones de la población española, medido por el número de años educativos, no ha ido acompañada por empleos de mayor calidad, por lo que el problema de la sobrecualificación ha adquirido cierta importancia. Esto implica que se deben promover mejoras en la calidad de los puestos de trabajo que se generen y reducir la dualidad del mercado laboral.

La situación del paro en nuestro país, derivada de la crisis financiera y del modelo de crecimiento basado fundamentalmente en la construcción, es insistenible socialmente. El empeoramiento de los resultados del mercado laboral ha

\footnotetext{
${ }^{5}$ Datos de EPA correspondientes al segundo trimestre. Con los datos más actuales de la EPA (cuarto trimestre de 2011), el número de ocupados es de 17.807.500, volviendo a niveles de empleo de la primera mitad de 2004, destruyendo más empleo y de forma más rápida que las economías europeas, situándose la tasa de paro en el 22,85\%. Para un análisis más detallado del mercado de trabajo en España desde 2007 se puede consultar Laborda (2011).

${ }^{6}$ Diversos trabajos, como Izquierdo y Lacuesta (2010) o Pérez (2010) señalan cierta relevancia del efecto del trabajador añadido en este período de crisis, derivado del aumento de la participación, sobre todo femenina, para intentar mantener los ingresos del hogar, dada la fuerte destrucción de empleo masculino, a diferencia de otras fases recesivas anteriores, con una presencia absoluta del efecto del trabajador desanimado.

${ }^{7}$ A lo largo del periodo de análisis el uso frecuente de estos contratos es un signo de una alta rotación laboral, con repercusiones negativas sobre el capital humano y la productividad de los trabajadores. Para su examen, ver Toharia (2005).
} 
desembocado en una elevada tasa de desempleo juvenil y en un notable incremento del desempleo de larga duración, afectando especialmente a determinados colectivos como los trabajadores poco cualificados o los mayores de 55 años. En este contexto, el adecuado diseño y la gestión de las políticas encaminadas a mejorar las cifras del mercado de trabajo, sobre todo, las políticas activas, son imprescindibles si se desean alcanzar resultados positivos de dicha intervención.

Dado el contexto actual, para mejorar la situación se apuesta por un cambio de modelo productivo que permita acercarnos a los países de nuestro entorno y conseguir reducir la tasa de paro, iniciando así la recuperación esperada. Entre las medidas para recuperar la economía y hacerla más competitiva, la Organización Internacional del Trabajo (OIT) (2011a) señala la necesidad de disminuir la temporalidad, promoviendo la creación de más y mejores puestos de trabajo, esto es, empleos de calidad. Considera que para mejorar nuestra competitividad se debe mejorar la calidad del empleo, por tanto este objetivo debe estar en el centro del debate de los distintos agentes sociales.

La importancia de determinados aspectos del trabajo como la remuneración, la jornada y su distribución, la autonomía laboral, las condiciones físicas, los riesgos en el puesto de trabajo o el entorno sociolaboral influyen de forma notable en la calidad del empleo. Se advierten, además, determinadas realidades que preocupan en el sentido de mermar la calidad del empleo, como el ya citado uso excesivo de la temporalidad, la dificultad para la conciliación de la vida laboral y familiar o un incremento de la competencia en un contexto de globalización. A la destrucción de empleo a lo largo de estos últimos años, hay que sumar la situación de inseguridad que tienen muchos trabajadores, con los efectos que ello puede tener en su salud y su bienestar, aspectos que influyen en la calidad del empleo.

\section{EL CONCEPTO DE CALIDAD DEL EMPLEO Y SUS COMPONENTES}

El marco conceptual de calidad del empleo resulta impreciso debido al carácter relativamente complejo y multidimensional que encierra. Cuando nos referimos a calidad estamos dando una connotación positiva a un hecho, además de percibirse como una situación anhelada. La calidad es un atributo que resulta de evaluar y calificar positivamente, en nuestro caso, el empleo y tendrá más o menos calidad en función de unos resultados o unas certezas. Por ello, cuando se describe la calidad del trabajo, en realidad lo que realizamos es una evaluación y emitimos una calificación que va a depender de un entorno económicosocial y político que condicionará la definición de dicho término.

La Comisión Europea aprueba en 2001 un marco para invertir en calidad, considerando que la calidad del empleo está integrada por diez dimensiones, 
cada una de las cuales está cuantificada por una serie de indicadores claves y contextuales. Estas dimensiones hacen referencia desde las características intrínsecas del trabajo, donde se incluye la satisfacción laboral, el salario, la jornada laboral, la formación del trabajador, el contenido del trabajo, la adecuación entre las dos anteriores, las posibilidades de promoción, hasta aquellas otras que aluden al trabajo y al contexto del mercado laboral con mención a aspectos como la igualdad de género, la salud y la seguridad, la flexibilidad, la accesibilidad al mercado de trabajo, la conciliación entre la vida laboral y familiar, el diálogo social y la participación de los trabajadores (Peña-Casas, 2009: 57). Para algunos autores este marco conceptual resulta impreciso. La falta de referencia explícita a teorías económicas o sociales hace que las críticas arrecien por considerar estas dimensiones criterios políticos subordinados a una serie de objetivos por conseguir (Dahl et al., 2009: 33). Así, en el informe Empleo en Europa 2008, la Comisión Europea manifiesta las dificultades que plantea la definición y contempla su modificación, de tal forma esto es así que estudios realizados sobre el significado del concepto de calidad del empleo proporcionado por la Unión Europea concluyen que "no había una sino múltiples y variadas definiciones" dependiendo de quién lo propone (Prieto et al., 2009: 210).

En términos generales, la calidad laboral se concibe como un atributo que se recomienda, siendo ambicionado y conveniente tanto social como políticamente en un marco económico. Y dos son los factores que concurren generando contextos complejos donde se favorece una mayor o menor facilidad para la existencia de la calidad del empleo frente a la cantidad del mismo; éstos son los mercados laborales (internos o externos, globales o locales, sectoriales o intersectoriales) y los ciclos económicos (de expansión y/o contracción de los mercados).

La actual crisis económica se dibuja como uno de los principales inconvenientes para la calidad. Por un lado, el ámbito del mercado de trabajo está en retracción desde el punto de vista de la demanda y, por otro lado, la principal preocupación en estos momentos es el incremento de la productividad ante una fuerte competencia en los mercados, circunstancia que tiende a relegar a un segundo plano el tema que nos ocupa (Prieto et al., 2009: 105).

En la actual situación debemos abordar el concepto de calidad del empleo desde dimensiones más amplias para ver su evolución temporal y así, no solo nos referimos a la acepción que abarca las características del empleo y las condiciones de trabajo (Somarriba et al., 2010; Ramos et al., 2010), sino también introduciendo las relaciones socioeconómicas que causan los actores que intervienen en el planteamiento de la calidad del empleo, es decir, las relaciones laborales y las políticas sociales. De esta forma, la calidad del empleo se refiere tanto al ámbito del lugar de trabajo concreto como a todo aquello que directa o 
indirectamente le rodea o interacciona con éste (Prieto et al., 2009: 98-103). La idea de análisis desde una perspectiva de diferentes calidades ha sido realizada por Reygadas (2002: 254-259) ${ }^{8}$ quien pretende unificar una concepción empresarial, cuyo objetivo sería el mantenimiento de la competitividad, con una concepción del trabajador que la vincula a un trato digno, a autonomía en la actividad laboral y a mejores salarios y prestaciones. Esta clasificación es tomada como punto de partida por Lahera (2006) que considera que el modelo de trabajo del futuro que diseñó la Unión Europea abarca explícita o implícitamente otras calidades: la calidad de las condiciones de trabajo, la calidad de las condiciones de empleo, la calidad de las relaciones laborales o la calidad de las políticas sociales.

Este trabajo toma como punto de referencia la anterior clasificación para poder abarcar el estudio de la calidad del empleo que, en su acepción más amplia, abarca las características del empleo y del puesto de trabajo (jornada, sueldo, cualificación necesaria,...), del entorno general del puesto y del mercado de trabajo (condiciones de trabajo, formación, diálogo social,...), de la negociación y participación de los agentes sociales y los trabajadores en la actividad económica y productiva (diálogo social, negociación colectiva, conocimiento de la organización...) y en los niveles de relación y protección social a los ciudadanos. Pero además, el atributo de calidad laboral depende de las circunstancias y experiencias particulares de cada uno. Un mismo trabajo o condición laboral tiene una calidad u otra en función del tipo de trabajo, del lugar del empleo y la situación particular con la que el trabajador percibe la realidad. Por ello, debemos tener en cuenta en nuestro análisis que la percepción o valoración subjetiva del individuo relativa a su puesto de trabajo (satisfacción laboral, expectativas laborales, etc.) es un factor a considerar. Esta combinación de variables objetivas y subjetivas dificulta la medición, aunque enriquece de forma importante el análisis.

A partir del concepto de calidad propuesto y desde una óptica multidimensional, se estudian los procesos y factores sociales que más directamente inciden en la calidad laboral por Comunidades Autónomas. Qué se evalúa, cómo y para qué son cuestiones que intentamos despejar.

\section{SELECCIÓN Y ELABORACIÓN DE LOS INDICADORES SIMPLES}

Este estudio de calidad del trabajo en el ámbito de las Comunidades Autónomas se lleva a cabo a través del enfoque de los indicadores sociales. Por ello, es

\footnotetext{
${ }^{8}$ Describe cinco asociaciones significativas de la idea de calidad a saber: calidad/prestigio, cali$\mathrm{dad} /$ eficacia, calidad/remuneración, calidad/trato digno y calidad/autonomía. Cada una de ellas las relaciona con otros aspectos del trabajo (Reygadas, 2002: 253)
} 
preciso seleccionar y construir un sistema de indicadores simples o parciales que sirvan para evaluar desde una óptica temporal el nivel de la calidad laboral de las distintas Comunidades Autónomas.

Tabla 1

Variables seleccionadas para el cálculo del indicador sintético $\mathrm{DP}_{2}$ organizadas por dimensiones

\begin{tabular}{|c|c|c|c|}
\hline Condiciones de Empleo & Condiciones de Trabajo & Relaciones Laborales & Política Social \\
\hline $\begin{array}{l}\text { - Satisfacción con el } \\
\text { salario } \\
\text { - Tasa de temporalidad } \\
\text { - Tasa de rotación anual } \\
\text { - Tasa de rotación } \\
\text { trimestral } \\
\text { - Tasa de parcialidad } \\
\text { - Involuntariedad del } \\
\text { trabajo a tiempo parcial } \\
\text { - Jornada laboral } \\
\text { - Coste salarial por } \\
\text { trabajador }\end{array}$ & $\begin{array}{l}\text { - Satisfacción con el entorno } \\
\text { físico } \\
\text { - Satisfacción con el ambiente de } \\
\text { trabajo } \\
\text { - Satisfacción con el tiempo de } \\
\text { descanso } \\
\text { - Satisfacción con la salud y la } \\
\text { seguridad } \\
\text { - Satisfacción con la autonomía } \\
\text { e independencia en el trabajo } \\
\text { - Percepción de estrés en el } \\
\text { trabajo } \\
\text { - Percepción de monotonía y } \\
\text { aburrimiento en el trabajo } \\
\text { - Percepción de situación de } \\
\text { riesgo en el trabajo } \\
\text { - Trabajo en equipo } \\
\text { - Protección y prevención de las } \\
\text { situaciones laborales de peligro } \\
\text { - Adecuación de la formación al } \\
\text { puesto que ocupa } \\
\text { - Nivel de estudios de } \\
\text { secundaria } \\
\text { - Participación en cursos de } \\
\text { formación ofrecidos por la } \\
\text { empresa }\end{array}$ & $\begin{array}{l}\text { - Existencia de } \\
\text { estructura que facilita } \\
\text { la negociación } \\
\text { colectiva } \\
\text { - Convenio o estatuto } \\
\text { especifico de } \\
\text { regulación } \\
\text { - Satisfacción con el } \\
\text { convenio colectivo } \\
\text { - Conocimiento de la } \\
\text { organización de la } \\
\text { empresa } \\
\text { - Conocimiento de los } \\
\text { objetivos de la } \\
\text { empresa } \\
\text { - Participación en toma } \\
\text { de decisiones }\end{array}$ & $\begin{array}{l}\text { - Formación } \\
\text { Ocupacional } \\
\text { - Cobertura por } \\
\text { desempleo } \\
\text { - Tasa de paro de } \\
\text { mayores de } 55 \\
\text { - Tasa de paro juvenil } \\
\text { - Tasa de desempleo } \\
\text { de larga duración } \\
\text { - Tasa de paro } \\
\text { femenina } \\
\text { - Tasa de paro total }\end{array}$ \\
\hline
\end{tabular}

Fuente: Elaboración propia.

En la Tabla 1 se recogen los 35 indicadores parciales utilizados, cuya información procede fundamentalmente de dos fuentes estadísticas: la Encuesta de Población Activa (EPA) y la Encuesta de Calidad de Vida en el Trabajo (ECVT). Como es habitual cuando se aplica la metodología de los indicadores sociales, los indicadores simples se presentan agrupados en distintos ámbitos, también denominados campos, componentes, dimensiones, áreas, etc. acorde con nuestra definición de calidad del trabajo previamente establecida. En este caso, se ha utilizado fundamentalmente una clasificación (Lahera, 2006; Somarriba et al., 2010) cuyos ámbitos considerados son: Condiciones de Empleo, Condiciones de Trabajo, Relaciones Laborales y Politica Social. A los indicadores pertenecientes a esta categorización se ha añadido el indicador sim- 
ple denominado satisfacción con el trabajo ${ }^{9}$ procedente de la ECVT y que no aparece encuadrado en ninguna de los ámbitos considerados, por tener un carácter más global.

Es preciso aclarar que en lo que concierne al presente trabajo, la agrupación en ámbitos o dimensiones es meramente orientativa, pues igualmente podría haberse hecho otra, ya que dada la metodología utilizada en esta investigación, la agrupación en componentes no condiciona ni el cálculo ni los resultados del indicador sintético, es decir, lo relevante es el conjunto de indicadores simples utilizados, independientemente de cómo estén agrupados. Este enfoque se adopta en trabajos como Zarzosa (1996, 2005) o Zarzosa y Somarriba (2012).

\section{AGREGACIÓN Y PONDERACIÓN DE LOS INDICADORES SIMPLES: METODOLOGÍA DEL INDICADOR SINTÉTICO DE DISTANCIA DE PENA}

En este epígrafe se presenta la metodología adoptada en la elaboración del Indicador Sintético de Calidad del Trabajo Dinámico que se emplea en esta investigación para aproximarse a la medición de la calidad del trabajo por Comunidades Autónomas. Se pretende obtener un indicador sintético que evalúe la evolución del nivel de calidad del trabajo regional, analizando el impacto que la crisis económica ha tenido en el periodo objeto de estudio. Para ello, resulta adecuado un indicador perteneciente a la familia de indicadores sintéticos "de distancia", concebido expresamente para medir distancias entre distintas situaciones o instantes temporales.

\subsection{Definición y propiedades del indicador sintético de distancia $D_{P_{2}}$}

La medida $\mathrm{DP}_{2}$, definida por Pena (1977), es un indicador sintético que agrega la información contenida en un conjunto de indicadores sociales y que está diseñada para realizar comparaciones interespaciales e intertemporales. Si tal y como se ha comentado anteriormente, se asume que el concepto de calidad del trabajo goza de un carácter multidimensional, se hace necesario sintetizar los diferentes estados o componentes que integran nuestro concepto por medio de lo que se denomina indicador sintético ${ }^{10}$.

En este caso, se toma como base de referencia ficticia una Comunidad Autónoma teórica, que alcanza los mejores valores de las variables objeto de estudio. $\mathrm{El}$ indicador $\mathrm{DP}_{2}$ devolverá las distancias de cada Comunidad respecto a esas

\footnotetext{
${ }^{9}$ Porcentaje de trabajadores que están bastante o muy satisfechos con su trabajo (ECVT).

${ }^{10}$ Para un estudio exhaustivo del indicador $\mathrm{DP}_{2}$, se puede consultar Pena (1977) y, posteriormente, Zarzosa (1996), Zarzosa (2005) y Somarriba (2008). Aquí solamente se presentará la metodología de manera breve, con el fin de suministrar los fundamentos metodológicos de la aplicación realizada (Zarzosa y Somarriba, 2012).
} 
Comunidad Autónoma teórica de referencia; por ello un valor más elevado de la medida de distancia estará indicando que estamos más alejados de ese valor máximo de calidad del empleo.

Se propone calcular un único indicador sintético en el que intervienen todas las unidades territoriales, en los tres instantes de tiempo, como si fueran un único conjunto de observaciones, de forma que cada unidad territorial se considera diferente para cada período.

La definición del indicador es:

Sean

- $\quad m$ el número de CAA

- $n$ el número de variables

- $\quad \mathrm{x}_{i j}$, el valor de la variable $i$ en la Comunidad $j$

- $\sigma_{i}$ la desviación típica de la variable $\mathrm{i}$

- $\quad R_{i, i-1, \ldots, 1}^{2}$ el coeficiente de determinación en la regresión de $X_{i}$ sobre $X_{i-1}$, $X_{i-2}, \ldots, X_{I}$

- Se define la distancia $\mathrm{P}_{2}$ de la siguiente forma:

$$
D P_{2}=\sum\left\{\left(d_{i} / \sigma_{i}\right)\left(1-R_{i, i-1, \ldots, 1}^{2}\right)\right\}
$$

con $R_{1}^{2}=0$, donde

$$
d_{i}=d_{i}\left(r^{*}\right)=\left|x_{r i}-x_{*_{i}}\right|
$$

y la base de referencia es

$$
X_{*}=\left(x_{*_{1}}, x_{*_{2}}, \ldots, x_{*_{n}}\right)
$$

Este indicador presenta como ventaja la resolución de un gran número de problemas: la agregación de variables expresadas en distintas medidas, la ponderación arbitraria y la duplicidad de información. Ello es posible con este método de distancia, ya que el orden de entrada de los indicadores parciales, que condiciona el peso relativo de cada variable, se determina a través de un algoritmo que alcanza la convergencia cuando el indicador cumple una serie de propiedades deseables. Además, por medio de un mecanismo corrector, tan sólo se retiene de cada variable la información nueva que ésta incorpora, aprovechando la información útil y evitando la información duplicada ${ }^{11}$.

${ }^{11}$ Sobre la comparación entre el indicador $\mathrm{DP}_{2}$ y otros procedimientos metodológicos de obténción de indicadores sintéticos de bienestar y conceptos afines puede consultarse los trabajos de Somarriba (2008) y Somarriba y Pena (2009a). 
A raíz del cumplimiento de una serie de propiedades del indicador sintético, ciertas variables cuyo aumento implican empeoramiento de la calidad del trabajo se multiplican por -1 de forma que un aumento del valor de cualquier variable suponga una mejora de la calidad. Se propone calcular un único indicador sintético en el que intervienen todas las unidades territoriales en los tres instantes de tiempo, como si fueran un único conjunto de observaciones, de forma que cada unidad territorial se considera diferente para cada período (Somarriba y Pena, 2011).

Las propiedades que verifica el indicador sintético de distancia $\mathrm{DP}_{2}$ hacen que éste sea una medida idónea para el objetivo planteado en la presente investigación. Dichas propiedades se enuncian a continuación de forma breve:

I. Existencia y Determinación: Dada la función matemática que define al indicador sintético $\mathrm{DP}_{2}$, es claro que éste existe y toma un valor determinado siempre que exista la varianza de todos y cada uno de los componentes y sea finita y distinta de cero.

II. Monotonía: El indicador sintético $\mathrm{DP}_{2}$ responde positivamente a una modificación positiva de las variables y negativamente a una modificación negativa.

III. Unicidad: Dado un conjunto ordenado de indicadores simples, la función matemática que define el indicador sintético $\mathrm{DP}_{2}$ proporciona un único resultado numérico.

IV. Invarianza: El indicador sintético es invariante a cambios de origen y/o escala en las medidas de los componentes.

V. Homogeneidad: El indicador sintético $\mathrm{DP}_{2}$ es una función homogénea de grado 1 respecto a los indicadores simples.

VI. Transitividad: Si (a), (b) y (c) son tres situaciones distintas del objetivo medido por el indicador sintético, y $\mathrm{DP}_{2}(\mathrm{a}), \mathrm{DP}_{2}$ (b) y $\mathrm{DP}_{2}$ (c) son los valores del indicador sintético correspondientes a esas tres situaciones se verifica que:

$$
\left.\begin{array}{l}
D P_{2}(a)>D P_{2}(b) \\
D P_{2}(b)>D P_{2}(c)
\end{array}\right\} \Rightarrow D P_{2}(a)>D P_{2}(c)
$$

VII. Exhaustividad: El indicador sintético debe ser tal que aproveche al máximo y de forma útil la información suministrada por los indicadores simples.

VIII. Aditividad: El indicador sintético de cada Comunidad Autónoma debe verificar los postulados I al VII. Además la diferencia entre dos indicadores sintéticos de dos regiones debe ser igual al indicador sintético que se obtendría comparando directamente las dos regiones. No obstante, Zarzosa $(1994,1996)$ demuestra que en general no se verifica que: 


$$
D P_{2}(r)-D P_{2}(k)=D P_{2}(r, k)
$$

Esto significa que la distancia $\mathrm{DP}_{2}$ no verifica estrictamente la propiedad de aditividad. Sin embargo, Zarzosa demuestra que la relación que existe entre ambas es:

$$
\left|D P_{2}(r)-D P_{2}(k)\right| \leq D P_{2}(r, k)
$$

IX. Invarianza respecto a la base de referencia: En Pena $(1977,102)$ siguiendo a Ivanovic se demuestra que si la base de referencia es la misma para las dos unidades y siempre que, para cada variable tome el valor máximo o uno superior a éste, o el valor mínimo o uno inferior a éste de la serie de valores de dicha variable, la distancia $\mathrm{DP}_{2}$ entre dos unidades, tanto calculada directamente como calculada mediante diferencia de dos distancias, no varía cualquiera que sea el vector de referencia.

X. Conformidad: Dado que el factor $\left(1-R_{i, i-1, i-2, \ldots, 1}^{2}\right)$ varía según el orden de entrada de las componentes; esto va a ocasionar que el resultado de la distancia $\mathrm{DP}_{2}$ varíe también en función del orden de entrada de dichas componentes y se hace necesario fijar un método de jerarquización de las componentes.

El criterio más lógico de ordenación es atender a la cantidad de información que cada componente aporta al indicador sintético, es decir, entraría en primer lugar aquella componente que contuviera mayor cantidad de información sobre el objetivo a medir y así sucesivamente. Se puede admitir, tal y como establece Pena, que la importancia de cada componente viene medida por el grado de dependencia entre él y el objetivo a medir, entonces la jerarquización de componentes se haría ordenándolos de mayor a menor según el valor absoluto del coeficiente de correlación simple entre cada componente y la $\mathrm{DP}_{2}$; es decir, si

$$
\left|r\left(D P_{2}, I_{i}\right)\right|>\left|r\left(D P_{2}, I_{h}\right)\right|
$$

entonces el componente $i$ contiene más información que $h$, por lo tanto $i$ entra antes que $h$.

En conclusión, el orden de entrada de las variables en el cálculo de la $\mathrm{DP}_{2}$ debe ser tal que los coeficientes de correlación, en términos absolutos, entre el indicador sintético resultante y los indicadores simples que lo componen estén ordenados de mayor a menor. Es decir, $D P_{2}=D P_{2}\left(I_{1}, I_{2}, \ldots, I_{n}\right)$ donde:

$$
\left|r\left(D P_{2}, I_{1}\right)\right|>\left|r\left(D P_{2}, I_{2}\right)\right|>\cdots>\left|r\left(D P_{2}, I_{n}\right)\right|
$$

A esta propiedad Zarzosa la denomina de conformidad. 
XI. No arbitrariedad en la importancia atribuida a los indicadores simples (Neutralidad). Esta propiedad fue introducida por Zarzosa (1996). Una de las críticas más habituales al diseño de los indicadores sintéticos es que las ponderaciones que se les atribuye a los indicadores simples y que reflejan su importancia suelen en la mayoría de los procedimientos ser arbitrarias. En el caso de la $\mathrm{DP}_{2}$, ésta no determina de antemano la importancia de los distintos indicadores parciales sino que su orden jerárquico es el resultado del procedimiento de cálculo. Las ponderaciones vienen determinadas, por un lado, por el factor corrector que elimina la información redundante, reteniendo sólo aquella información que resulta útil $\mathrm{y}$, por otro, por los valores absolutos de los coeficientes de correlación entre cada indicador parcial y el indicador sintético, que determinan el orden utilizado en la estimación de las regresiones y, por tanto, en el cálculo del indicador sintético.

\section{FACTORES EXPLICATIVOS DE LA CALIDAD DEL TRABAJO}

Una de las principales ventajas de emplear la metodología de la $\mathrm{DP}_{2}$ en el análisis de la calidad del trabajo es la posibilidad de estudiar la repercusión que, con respecto a los demás, tiene cada indicador simple en la determinación de los resultados. A continuación, se analiza la importancia relativa de cada indicador simple en la explicación de las disparidades de la calidad del trabajo en el marco de las Comunidades Autónomas, por medio de la correlación lineal absoluta con el indicador sintético y por medio del factor corrector.

Como se ha explicado en el apartado anterior, el valor absoluto del coeficiente de correlación lineal es la medida utilizada para jerarquizar los indicadores simples en las distintas iteraciones de cálculo del indicador sintético. Y además, como ya se ha comentado se ha procedido a calcular un único indicador sintético, en el que intervienen todas las Comunidades Autónomas en los tres años de referencia, por ello la estructura del indicador es común para los tres años.

En la Tabla 2 los indicadores simples aparecen ordenados según el grado de correlación absoluta con el ISCTD. El primer indicador simple, satisfacción con la salud y la seguridad, es el que presenta una mayor correlación lineal en valor absoluto con el indicador final, y el último indicador simple (jornada) el que cuenta con la menor correlación absoluta.

Dado que el indicador satisfacción con la salud y la seguridad es el indicador parcial más correlacionado con el indicador sintético, incorpora toda su información, por eso su correspondiente factor corrector es la unidad. La percepción de estrés en el trabajo es el segundo indicador parcial en intervenir en el cálculo del indicador sintético, dada su correlación absoluta, incorporando un $28 \%$ de su información, ya que el $72 \%$ restante resulta redundante con respecto a la 
información contenida en la satisfacción con la salud y la seguridad. Ambos indicadores reflejan aspectos relacionados con las condiciones de trabajo en las que los individuos desarrollan su actividad.

Tabla 2

Estructura del indicador ISCTD. 2000-2010

\begin{tabular}{|c|c|c|}
\hline Correlación absoluta & Variable & Factor corrector \\
\hline 0,689 & Satisfacción con la salud y la seguridad & 1 \\
\hline 0,5314 & Percepción de estrés en el trabajo & 0,28 \\
\hline 0,4964 & Involuntariedad del trabajo a tiempo parcial & 0,0761 \\
\hline 0,4949 & Formación ocupacional & 0,6267 \\
\hline 0,4948 & Satisfacción con el salario & 0,6282 \\
\hline 0,4692 & Satisfacción con el entorno físico & 0,1748 \\
\hline 0,467 & Cobertura por desempleo & 0,0765 \\
\hline 0,4642 & Percepción de monotonía y aburrimiento en el trabajo & 0,2657 \\
\hline 0,4502 & Tasa de parcialidad & 0,5145 \\
\hline 0,4303 & Protección y prevención de las situaciones laborales de peligro & 0,3197 \\
\hline 0,4199 & Satisfacción con el convenio colectivo & 0,6766 \\
\hline 0,4133 & Tasa de paro de mayores de 55 & 0,183 \\
\hline 0,4044 & Tasa paro total & 0,3452 \\
\hline 0,3603 & Adecuación de la formación al puesto que ocupa & 0,0586 \\
\hline 0,3566 & Tasa de paro juvenil & 0,4037 \\
\hline 0,3533 & Trabajo en equipo & 0,3642 \\
\hline 0,3307 & Percepción de situación de riesgo en el trabajo & 0,1138 \\
\hline 0,3077 & Satisfacción con el ambiente de trabajo & 0,3731 \\
\hline 0,2848 & Satisfacción con el tiempo de descanso & 0,1149 \\
\hline 0,2793 & Conocimiento de los objetivos de la empresa & 0,1459 \\
\hline 0,2789 & Participación en cursos de formación ofrecidos por la empresa & 0,124 \\
\hline 0,2433 & Coste salarial por trabajador & 0,1242 \\
\hline 0,2178 & Tasa de paro femenina & 0,1939 \\
\hline 0,1863 & Satisfacción con el trabajo & 0,2942 \\
\hline 0,1385 & Conocimiento de la organización de la empresa & 0,4915 \\
\hline 0,1005 & Tasa de desempleo de larga duración & 0,0766 \\
\hline 0,0984 & Tasa de temporalidad & 0,0776 \\
\hline 0,0949 & Existencia de estructura que facilita la negociación colectiva & 0,0944 \\
\hline 0,085 & Satisfacción con la autonomía e independencia en el trabajo & 0,0881 \\
\hline 0,0832 & Participación en toma de decisiones & 0,07 \\
\hline 0,071 & Convenio o estatuto especifico de regulación & 0,0623 \\
\hline 0,0689 & Tasa de rotación anual & 0,1404 \\
\hline 0,0671 & Tasa de rotación trimestral & 0,256 \\
\hline 0,0528 & Nivel de estudios de secundaria & 0,1261 \\
\hline 0,0166 & Jornada laboral & 0,0894 \\
\hline
\end{tabular}

Fuente: Elaboración propia. 
En cuanto a las dimensiones definidas previamente en nuestro concepto de calidad del trabajo, si nos fijamos en los indicadores simples que incorporan al indicador sintético elevados porcentajes de su información (superiores al 40\%), podemos concluir que todas ellas (condiciones de trabajo, condiciones de empleo, relaciones laborales y política social) se encuentran representadas. Recordemos que el indicador satisfacción con la salud y la seguridad incorporaba el $100 \%$ de su información al indicador sintético y pertenece a la dimensión condiciones de trabajo, satisfacción con el convenio colectivo $(67 \%)$ y conocimiento de la organización de la empresa (49\%) conciernen a la componente de relaciones laborales, la tasa de parcialidad (51\%) y la satisfacción con el salario (62\%) guardan relación con las condiciones de empleo y por último, la tasa de paro juvenil (40\%) y la formación ocupacional (62\%) están integradas dentro de política social.

En todo caso se observa un aumento de la importancia de los aspectos relacionados con el escenario donde el individuo desarrolla su tarea y como él los percibe, en relación tanto a los aspectos propiamente dichos del puesto como a los del entorno y con respecto a otros indicadores más tradicionales de carácter objetivo.

En cuanto al orden de entrada de las variables en el ISCTD, cabe señalar la importancia de indicadores simples de contenido subjetivo, ocupando seis de las doce primeras posiciones, incorporando altos porcentajes de información como la satisfacción con la salud y la seguridad, la satisfacción con el salario, la satisfacción con el convenio colectivo y la percepción de monotonía y de estrés en el trabajo.

Por otro lado, se advierte una menor relevancia de las variables que muestran la situación del contexto laboral, como las tasas de paro (total, femenina, mayores de 55 años y de larga duración), que están menos correlacionadas. También se aprecia que las variables relacionadas con la estabilidad laboral (tasa de temporalidad, tasa de rotación anual y trimestral) contribuyen con poca información al ISCTD.

Ahora bien, hay que tener en cuenta que un indicador simple puede ser muy importante para medir la calidad del trabajo y, sin embargo, no ser discriminante en el conjunto de las Comunidades Autónomas, de manera que no tiene gran influencia en las distancias entre esas Comunidades, por ejemplo la jornada laboral, a pesar de la importancia concedida por los trabajadores al tiempo de trabajo y su distribución.

\section{LA CALIDAD DEL TRABAJO EN LAS COMUNIDADES AUTÓNOMAS}

Aplicando la metodología de la medida $\mathrm{DP}_{2}$ a los indicadores simples de la Tabla 1, se han obtenido los resultados que aparecen en la Tabla 3, que recoge 
los valores del ISCTD en las distintas Comunidades Autónomas en los años 2000, 2007 y 2010. Además aparecen los rankings regionales en función del ISCTD en los tres años de estudio y la variación de éste del 2007 respecto al año 2000 y del 2010 respecto al 2000 y 2007. Tal como se definió la base de referencia, mayores valores del indicador sintético significan una mayor distancia al objetivo de una mejor calidad del trabajo. Esto es, una Comunidad Autónoma que presentara la mejor situación para todos los indicadores simples tendría un valor nulo del ISCTD y, por tanto, el máximo nivel de calidad del empleo.

Si bien es deseable tender hacia una mayor calidad laboral a largo plazo, durante el período de análisis 2000-2010 se produce un empeoramiento del indicador en todas las Comunidades Autónomas. No obstante, cabe destacar la importancia de las fases del ciclo económico en la determinación de los niveles de calidad del trabajo. En la fase de recesión se aprecia un empeoramiento casi generalizado del indicador con un incremento de las disparidades entre las Comunidades, mientras que en la fase expansiva se produce una reducción de las desigualdades regionales, a pesar del dispar comportamiento regional del ISCTD. En el año 2000 se advierte una gran dispersión en cuanto a los niveles de calidad laboral por autonomías, tendiendo a reducirse esas desigualdades en 2007 con una menor dispersión en los valores del Indicador Sintético, para volver a distanciarse en el 2010. Utilizando la diferencia entre el valor mínimo y el máximo de nuestro ISCTD como medida de dispersión, ésta pasa de superar los catorce puntos en el 2000, a reducirse a sólo cinco puntos en 2007, disparándose, en tan sólo tres años, a más de nueve puntos en 2010.

Este hecho observado nos hace plantear una presentación de los datos en función del ciclo económico que se marca en dos fases: una primera expansiva que abarca el periodo 2000-2007 y otra segunda de carácter recesivo que comprende el periodo 2007-2010.

I. En la fase expansiva (2000-2007) se produce una mejora del indicador en Canarias, Andalucía, Castilla y León y Extremadura, manteniéndose su valor en el País Vasco y Galicia. Los mejores resultados del ISCTD tanto en Canarias como en Extremadura permiten que estas dos Comunidades asciendan de forma notoria en el ranking, subiendo doce y ocho posiciones, alcanzando el cuarto y tercer lugar, respectivamente.

A la vista de la información de la matriz de indicadores simples ${ }^{12}$, se observa que en Canarias y Extremadura los principales factores que pueden estar detrás de su avance en el ISCTD son la mejora de determinadas variables de condi-

${ }^{12}$ Dado el tamaño tan amplio de la matriz (51 x 35), resulta imposible mostrar su contenido en este artículo. Por ello, los lectores interesados pueden solicitar dicha información por correo electrónico a los autores. 
ciones de trabajo como el aumento del porcentaje de los satisfechos con el entorno físico, con la autonomía e independencia en el trabajo o con la salud y la seguridad.

En cuanto a las condiciones de empleo, en estas dos regiones el porcentaje de satisfechos con el salario disminuye ${ }^{13}$ aunque muy ligeramente, y, por otro lado, se produce un elevado aumento del porcentaje de involuntariedad del trabajo a tiempo parcial (donde más ha aumentado, junto a Andalucía). También hay que considerar que a pesar de la disminución de los niveles de desempleo de las distintas categorías, ocupan en la mayoría de los casos los primeros lugares de la clasificación, por sus elevadas tasas de paro. En el caso de las variables de relaciones laborales, cabe señalar para el caso de Canarias el buen comportamiento de los indicadores sobre el conocimiento de la organización de la empresa y de los objetivos de la empresa.

A pesar del empeoramiento de su ISCTD, por un lado, Aragón y La Rioja son las dos regiones que se sitúan en las primeras posiciones, intercambiándose los lugares en los dos años y, por otro lado, Cantabria mantiene la misma posición, la quinta, tanto en 2000 como en 2007.

Si en el año 2000 Andalucía ocupa el último lugar en la clasificación, siete años más tarde esta región asciende dos puestos, estando la Comunidad Valenciana en la cola del ranking, por la pérdida de siete posiciones. Esta situación viene motivada principalmente por la disminución del porcentaje de satisfechos con el entorno físico y con la salud y la seguridad, el aumento de la percepción de estrés en el trabajo, una reducción de la importancia de la formación ocupacional y por los indicadores de las tasas de paro.

II. En la etapa que hemos denominado de crisis, la calidad laboral se ha visto empeorada en términos generales en todas las Comunidades Autónomas. Rompiendo la regla, en sólo tres regiones se advierten mejoras poco significativas, en torno a medio punto del ISCTD. Así, Navarra pasa de la decimocuarta posición en 2007 a la quinta en 2010, Cataluña de la octava a la cuarta y Cantabria consigue ascender a la primera posición con respecto al ranking del 2007, procedente del quinto puesto, y desbancando al segundo lugar a Aragón.

Cabe destacar el espectacular empeoramiento de Castilla y León, con un incremento del valor del indicador de prácticamente ocho puntos, Extremadura con un aumento de casi siete puntos y Andalucía y Canarias en torno a seis. Si nos detenemos en las razones de estos cambios observamos que existe una disminución en la percepción de los trabajadores respecto a las condiciones de trabajo, a pesar de existir una mejora en la formación. Pero lo realmente significativo es el importante incremento en las tasas de paro, en especial la total y la juvenil, alcanzando unos niveles bastante preocupantes.

\footnotetext{
${ }^{13} \mathrm{Al}$ igual que en el resto de Comunidades Autónomas, con la excepción de Galicia.
} 
A pesar del incremento del indicador sintético, es decir, del descenso de los niveles de calidad laboral, no se observan oscilaciones muy fuertes en Comunidades Autónomas como Castilla-La Mancha, Comunidad Valenciana, Asturias y Murcia. El hecho destacable es que dichas regiones, en comparación con las restantes, mejoran sus posiciones de forma importante. Estas oscilaciones en la posición del ranking no deben interpretarse como una mejora de la calidad del empleo en sus territorios, más bien debemos deducir que la falta de una variación significativa del valor del indicador da como resultado una mejor situación en el ranking derivado del empeoramiento de otras Comunidades. Como se puede apreciar en la Tabla 3, Asturias se sitúa en el mismo lugar en el ranking que ocupó en el 2000, a pesar de que su indicador aumenta, esto es, se produce un deterioro en su calidad del empleo con respecto a ese año.

Tabla 3

Ordenación de las Comunidades Autónomas españolas según el Indicador Sintético de Calidad del Trabajo Dinámico (ISCTD)

\begin{tabular}{|l|c|c|c|c|c|c|c|c|c|}
\hline & \multicolumn{3}{|c|}{$\mathrm{DP}_{\mathbf{2}}$} & \multicolumn{3}{c|}{ Ranking } & \multicolumn{3}{c|}{ Variación Ranking } \\
\hline & $\mathbf{2 0 0 0}$ & $\mathbf{2 0 0 7}$ & $\mathbf{2 0 1 0}$ & $\begin{array}{c}\text { Ranking } \\
\mathbf{2 0 0 0}\end{array}$ & $\begin{array}{c}\text { Ranking } \\
\mathbf{2 0 0 7}\end{array}$ & $\begin{array}{c}\text { Ranking } \\
\mathbf{2 0 1 0}\end{array}$ & $\mathbf{2 0 0 7 / 2 0 0 0}$ & $\mathbf{2 0 1 0 / 2 0 0 0}$ & $\mathbf{2 0 1 0 / 2 0 0 7}$ \\
\hline Andalucía & 23.63 & 21.91 & $\mathbf{2 7 . 6 9}$ & 17 & 15 & 16 & -2 & -1 & 1 \\
\hline Aragón & 15.18 & 17.20 & 19.89 & 2 & 1 & 2 & -1 & 0 & 1 \\
\hline Asturias & 16.12 & 19.68 & 20.03 & 3 & 6 & 3 & 3 & 0 & -3 \\
\hline Baleares & 16.55 & 20.84 & 23.28 & 4 & 9 & 10 & 5 & 6 & 1 \\
\hline Canarias & 22.37 & 19.31 & 25.09 & 16 & 4 & 15 & -12 & -1 & 11 \\
\hline Cantabria & 17.17 & 19.64 & 19.16 & 5 & 5 & 1 & 0 & -4 & -4 \\
\hline Castilla La Mancha & 18.77 & 21.55 & 21.68 & 8 & 12 & 7 & 4 & -1 & -5 \\
\hline Castilla y León & 21.90 & 20.90 & 28.47 & 15 & 10 & 17 & -5 & 2 & 7 \\
\hline Cataluña & 18.09 & 20.63 & 20.04 & 7 & 8 & 4 & 1 & -3 & -4 \\
\hline Comunitat Valenciana & 19.46 & 23.28 & 23.45 & 10 & 17 & 11 & 7 & 1 & -6 \\
\hline Extremadura & 19.86 & 18.67 & 25.06 & 11 & 3 & 14 & -8 & 3 & 11 \\
\hline Galicia & 21.28 & 21.21 & 25.05 & 13 & 11 & 13 & -2 & 0 & 2 \\
\hline Madrid & 21.68 & 22.76 & 24.47 & 14 & 16 & 12 & 2 & -2 & -4 \\
\hline Murcia & 19.23 & 21.62 & 22.96 & 9 & 13 & 9 & 4 & 0 & -4 \\
\hline Navarra & 17.66 & 21.78 & 20.15 & 6 & 14 & 5 & 8 & -1 & -9 \\
\hline País Vasco & 19.95 & 19.90 & 22.90 & 12 & 7 & 8 & -5 & -4 & 1 \\
\hline Rioja & 9.06 & 17.46 & 21.28 & 1 & 2 & 6 & 1 & 5 & 4 \\
\hline
\end{tabular}

Fuente: Elaboración propia.

Finalmente podemos observar de forma más clara la evolución que ha tenido la calidad laboral en las distintas Autonomías por medio de la elaboración de cartogramas para cada uno de los años de nuestro análisis. Las Comunidades Autónomas se han agrupado en cuatro niveles en función del recorrido total del indicador sintético en el periodo objeto de estudio. Una mayor intensidad de 
color se asocia a peores valores del ISCTD. Como se puede observar se ha producido un gran cambio en la imagen espacial en cuanto a la calidad laboral.

De esta manera, descubrimos la existencia de una gran dispersión en los niveles del ISCTD en el año 2000, habiendo regiones en todos los grupos. Nos situamos en un escenario de grandes desigualdades del ISCTD. Además se advierte que en la franja Norte se ubican con carácter general las regiones con una mayor calidad del trabajo.

Figura 1

Evolución Temporal del ISCTD 2000, 2007 y 2010

ISCTD 2000

ISCTD 2007
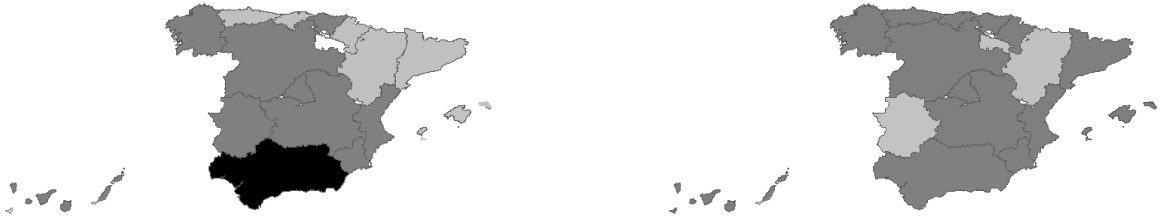

ISCTD2010
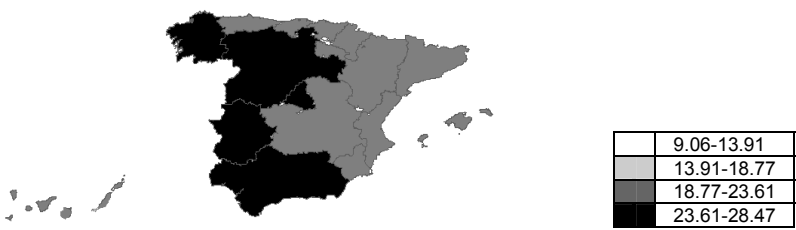

Fuente: Elaboración propia.

La buena situación económica en España en el período 2000-2007 se ha traducido en una mayor homogeneidad de los niveles de calidad entre las distintas Comunidades Autónomas, que están concentradas en los dos grupos intermedios. Solamente La Rioja, Aragón y Extremadura se encuentran en el segundo grupo. En relación con la figura anterior, se deduce que Extremadura experimenta un avance, mientras que La Rioja un retroceso en sus niveles de calidad.

Con los efectos de la crisis en pleno apogeo en el año 2010, se observa una gran zona negra dominando casi la mitad del mapa, signo del empeoramiento de la calidad del trabajo. Esta panorámica se traduce en una gran polarización espacial, en el que las Comunidades de la zona Norte-Este junto a los dos archipiélagos se enfrentan al Oeste-Sur. 


\section{CONCLUSIONES}

La aplicación del criterio de calidad a todo tipo de productos, servicios, actividades e instituciones es un hecho que se extiende en las últimas décadas, aunque la aparición de la problemática de la calidad del empleo tenga unos rasgos que le son propios. La evolución y la situación actual de la calidad laboral en España muestran unas peculiaridades derivadas fundamentalmente de los factores económico-sociales que inciden más directamente en el empleo.

Los resultados derivados del indicador ISCTD muestran que se ha hecho una buena selección de indicadores parciales acorde con el concepto de calidad del empleo que se ha considerado en este trabajo.

Cabe destacar la importancia de la fase del ciclo económico en el proceso de convergencia o divergencia de los niveles de calidad laboral entre las Comunidades Autónomas españolas. Si en la fase expansiva de la economía española 2000-2007, con mejoras en los niveles de calidad en seis Comunidades Autónomas, se advierte una reducción de las desigualdades regionales, en la fase de recesión 2007-2010, al empeoramiento del valor del ISCTD en casi todas las Comunidades, se suma una ampliación de las disparidades entre las Comunidades Autónomas.

Con los datos correspondientes a cada año analizado, la fotografía final en cuanto a calidad laboral permite hablar de una cierta polarización espacial a lo largo del período de estudio. Se advierte que las Comunidades situadas al Oeste y Sur se encuentran en peor posición en cuanto a calidad del empleo que las del Este y los archipiélagos, a las que se unen Asturias, Cantabria y el País Vasco.

Por otro lado, la relevancia en el ISCTD de las variables subjetivas, ocupando seis de las doce primeras posiciones, incorporando además un elevado porcentaje de información, pone de manifiesto la trascendencia de las percepciones de los individuos en su calidad laboral. La importancia de las valoraciones de los trabajadores hace que sea un elemento a tener en cuenta por los empresarios, ya que el bienestar de los trabajadores en su centro de trabajo se traduce en una mayor motivación y, por tanto, en una mayor productividad.

En la necesaria creación de empleo se hace imprescindible que junto a la cantidad exista un compromiso hacia la calidad laboral en un mercado en proceso de transformación. La mejora de la calidad del empleo no puede ser resultado de la intervención en una sola de las cuatro dimensiones que concurren en la configuración del concepto de calidad que hemos considerado. Así, la introducción de un conjunto de prácticas económicas requiere la transformación de políticas tanto sociolaborales como de relaciones laborales. Cualquier mejora de calidad del empleo pasa por introducir cambios en la regulación del trabajo que incremente la estabilidad laboral de los trabajadores frente al despido. Y esto requiere una regulación pública favorable a la mejora de calidad. 
Como reconoce la OIT (2011b), el crecimiento económico sin creación de empleo de calidad no es sostenible.

\section{REFERENCIAS BIBLIOGRÁFICAS}

COMISIÓN EUROPEA (2009): El empleo en Europa 2008, Madrid: Ministerio de Trabajo y Asuntos Sociales.

COMISIÓN EUROPEA (2010): "Europa 2020: Una estrategia para un crecimiento inteligente, sostenible e integrador", Bruselas: COM (2010)2020.

DAHL, S.; NESHEIM, T. Y OLSEN K, (2009): La calidad del trabajo. Concepto y medición. En M. Guillén, R. Gutiérrez, S. González, Calidad del trabajo en la Unión Europea. Concepto, Tensiones, Dimensiones (pp. 25-51). Pamplona: Thomson Civitas.

GREEN, F. (2009): "Job quality in Britain", en Praxis, 1: pp. 8-33.

IZQUIERDO, M. y LACUESTA, A. (2010): "Desarrollos recientes en el mercado de trabajo", en Papeles de Economía Española, 124: pp. 2-16.

LABORDA PERALTA, A. (2011): "El mercado laboral español: Situación y perspectivas a corto plazo", en Cuadernos de Mercado de Trabajo, 7: pp. 22-39.

LAHERA, A. (2006): "Diseñando el futuro del trabajo: ¿avanzando hacia un trabajo decente y un empleo de calidad o hacia un trabajo degradado y un empleo precarizado?" (pp. 365-405). En F. Vidal Fernández, La exclusión social y el estado de bienestar en España, Barcelona: Icaria.

MULAS-GRANADOS, C. y FERNÁNDEZ, R. (2011): "El empleo en España frente al reto de la Estrategia 2020", en Cuadernos de Mercado de Trabajo, 6: pp.7-15.

OIT (2011a): España. Empleos de calidad para una nueva economía, Ginebra: Instituto Internacional de Estudios Laborales.

OIT (2011b): Global Employment Trends, Ginebra: OIT.

PENA, J. B. (1977): Problemas de la medición del bienestar y conceptos afines (Una aplicación al caso español), Madrid: INE.

PEÑAS- CASAS, R. (2009): "Más y mejores empleos. Marcos conceptuales y políticos del trabajo y el empleo en la Unión Europea”. En M. Guillén, R. Gutiérrez, S. González, Calidad del trabajo en la Unión Europea. Concepto, Tensiones, Dimensiones (pp. 53-74) Pamplona: Thomson Civitas.

PÉREZ INFANTE, J.I. (2010), "Crisis y mercado de trabajo", Tribuna abierta de la Asociación Española de Economía del Trabajo (8 de noviembre de 2011). Disponible en: http://www.aeet.eu/es/tribuna-abierta/90-casi-cinco-millonesde-parados.html [5 de marzo de 2012]. 
PRIETO, C.; ARNAL, M.; CAPRILE, M. y POTRONY, J. (2009): La calidad del empleo en España: una aproximación teórica y empírica. Madrid: Ministerio de Trabajo e Inmigración.

RAMOS, G.; NEGRO, A.; MERINO, M.C. y SOMARRIBA, N. (2010): "El empleo en la Unión Europea: un análisis de la calidad del trabajo", en Revista de Relaciones Laborales, Lan Harremanak, 22: pp. 17-43.

REYGADAS, L. (2002): Ensamblando culturas. Diversidad y conflicto en la globalización de la industria. Barcelona, Gedisa.

SOMARRIBA, N. (2008): Aproximación a la medición de la calidad de vida en la Unión Europea. Doctoral Thesis, Resource document

http://www.eumed.net/tesis/2010/mnsa/index.htm Accessed July 2009.

SOMARRIBA, N. and PENA, B. (2009a): Synthetic Indicators of Quality of Life in Europe, in Social Indicators Research, doi 10.1007/s11205-008-9356-y

SOMARRIBA, N. y PENA, B. (2010): "Un análisis dinámico de la calidad de vida y de la convergencia en Europa", en Anales de estudios económicos y empresariales, 20: pp. 283-324.

SOMARRIBA, N.; MERINO, M.C.; RAMOS, G. y NEGRO, A. (2010): "La calidad del trabajo en la Unión Europea", en Estudios de Economía Aplicada, vol. 28-3: pp. 1-22.

TOHARIA, L. (dir.) (2005): El problema de la temporalidad en España: un diagnóstico, Madrid: Ministerio de Trabajo y Asuntos Sociales.

ZARZOSA, P. (1996): Aproximación a la medición del bienestar social. Valladolid: Universidad de Valladolid.

ZARZOSA, P. (dir). (2005): La calidad de vida en los municipios de la provincia de Valladolid, Valladolid: Diputación de Valladolid.

ZARZOSA, P. y SOMARRIBA, N. (2012): "An assessment of Social Welfare in Spain: Territorial analysis using a Synthetic Welfare Indicator", in Social Indicators Research, d.o.i. 10.1007/s11205-012-0005-0. 